\title{
Acute airway management
}

\author{
Nikhil Panda, Dean M. Donahue \\ Division of Thoracic Surgery, Department of Surgery, Massachusetts General Hospital, Boston, MA, USA \\ Correspondence to: Dean M. Donahue, MD. Massachusetts General Hospital, 55 Fruit Street, Blake 1570, Boston, MA 02114, USA. \\ Email: ddonahue@mgh.harvard.edu.
}

\begin{abstract}
Acute airway management has challenged clinicians for nearly four millennia. History underscores the discoveries of surgeons and anesthesiologists, whose advances in technology and surgical technique have transformed management algorithms from primarily surgical tracheostomy, to transoral endotracheal intubation under direct laryngoscopy. Despite this progress and a better understanding of airway anatomy, physiology and pathogenesis of disease, the acute airway, whether obstructed, traumatically disrupted, or externally compressed, remains a life-threatening challenge. The role of all clinicians in acute airway management is patient stability and emergent control of the airway to ensure patency as well as adequate oxygenation and ventilation. The standard of care remains transoral intubation under direct laryngoscopy with use of indirect laryngoscopy as a first adjunct. If unsuccessful, surgical intubation of the trachea via cricothyroidotomy with subsequent conversion to open tracheostomy is the procedure of choice. While there is growing support for the use of percutaneous tracheostomy as an alternative to surgical intubation of the trachea after failed transoral intubation, the potential for damage to critical neck structures and longer timeto-intubation must be considered. In this perspective, we provide a history of acute airway management, highlighting milestones in the fields of airway surgery and anesthesia. We present a review of current medical and surgical approaches to managing the acute airway, including the risks, benefits and appropriateness of each approach with respect to patient stability, available equipment, clinician training and patient outcomes. We conclude with an emphasis on the role of the thoracic surgeon in prevention and the critical nature of regular surveillance of patients with chronic, partial tracheal obstruction.
\end{abstract}

Keywords: Airway obstruction; surgical cricothyroidotomy; percutaneous tracheostomy; post-intubation tracheal stenosis

Submitted Nov 28, 2017. Accepted for publication Dec 21, 2017.

doi: 10.21037/acs.2018.01.15

View this article at: http://dx.doi.org/10.21037/acs.2018.01.15

\section{Introduction}

The acute airway, whether obstructed, traumatically disrupted, or externally compressed, requires emergent attention. Reports of acute airway management date back to 2000 BC (1). Despite further understanding of anatomy, physiology and pathogenesis of disease, management of the acute airway remains a challenge. The acute airway may be encountered in the pre-hospital setting, within the emergency ward, intensive care units and operating rooms. Physicians at various stages of clinical development may be called upon to manage the acute airway. Furthermore, advances in technology and anesthesia have armed a larger body of clinicians with the ability to address the acute airway.

The thoracic surgeon in particular has an additional responsibility in the management of the acute airway: prevention. Post-intubation tracheal stenosis and malignant airway lesions remain the most common pathologies of the tracheobronchial tree that may progress to life-threatening airway obstruction, if not properly treated and surveilled (2). Thoracic surgeons are therefore charged with appropriate and diligent management and follow-up of these patients.

In this perspective, we provide a history of acute airway management and present a review of current non-surgical 
and surgical approaches. We discuss the pros and cons of various treatments with our own perspectives as thoracic surgeons and trainees and highlight our role in prevention.

\section{Historical perspective}

The first descriptions of acute airway management date back to 2000 BC in ancient Hindu and Egyptian texts (1). While many have described various techniques of acute airway management since this time, including Alexander the Great's battle-sword tracheotomy on a soldier with an upper airway obstruction, the first human series was described by $19^{\text {th }}$ century French internist, Dr. Armand Trousseau $(3,4)$. As a clinician during the diphtheria pandemic, he routinely performed open surgical tracheostomy on patients with diphtheria-related upper airway obstruction. During the same era were also the first reports of endotracheal intubation, which were often blindly placed transoral or transnasal tubes in patients undergoing elective surgery.

The use of endotracheal intubation, rather than the surgical tracheostomy, is the result of scientific advancements in two areas of medicine: direct laryngoscopy and anesthesia. In 1913, Dr. Chevalier Jackson performed the first tracheal intubation using direct laryngoscopy, visualizing the passage of the tube through the vocal cords (5). His laryngoscope, notably, inspired subsequent designs by Drs. Robert Miller and Robert Macintosh, both of whom remain known today for their named laryngoscope blades (6). The first use of anesthesia occurred in Boston at Massachusetts General Hospital in 1846, where dentist Dr. William Morton demonstrated the use of ether (7). Though a potent anesthetic, ether does not depress the respiratory drive and therefore endotracheal intubation is not required. It was not until the introduction of chemical paralysis in the mid- $20^{\text {th }}$ century that endotracheal intubation became routine (8). As a result, surgeons and anesthesiologists became armed with an alternative technique to surgical tracheostomy for airway management and today, nearly four millennia since the first report of acute airway management, endotracheal intubation with direct laryngoscopy has become the gold standard for securing the airway.

When endotracheal intubation fails, surgical intubation of the trachea via cricothyroidotomy with subsequent conversion to open tracheostomy is the standard of care. Recently, authors have described the use of percutaneous tracheostomy as a means of emergency airway management (9). While first described by Dr. Pasquale Ciaglia in 1985 as an alternative to elective surgical tracheostomy for patients requiring prolonged endotracheal intubation, it was not until 2004 when percutaneous tracheostomy was employed for emergency airway management after failed endotracheal intubation (10). Its use in this setting remains controversial, as discussed later in this perspective.

The history of acute airway management highlights landmarks within the fields of airway surgery and anesthesiology. Likewise, innovations in technology have allowed for a greater number of successful intubations, fewer emergency surgical airways, as well as more effective teaching for trainees. All clinicians should be aware of this history when understanding the management of the acute airway.

\section{Assessment of the patient with acute airway compromise}

The priorities of the thoracic surgeon in managing a patient with an acutely compromised airway, regardless of the etiology, include maintaining a patent airway, allowing adequate oxygenation and ventilation and minimizing harm during airway placement. If rapid deterioration is unlikely, the etiology and location of airway compromise, as well as concurrent medical problems, may be assessed while closely monitoring the patient.

The most common cause of acute airway compromise is airway obstruction (11). This may be from central loss of respiratory drive, as in the comatose patient, leading to collapse of soft tissues over the airway, or else primary obstructing or secondary compressing processes of the airway. Primary processes include inflammation, as in anaphylaxis, infectious, as in acute epiglottitis, or foreignbody inhalation (12). Secondary processes include adjacent neck trauma with airway obstruction from expanding hematoma, compression from head and neck cancers, or rare cases of bilateral recurrent laryngeal nerve injuries leading to bilateral vocal cord paralysis (13).

The thoracic surgeon should recognize chronic conditions which have the potential to obstruct the airway, including post-intubation tracheal stenosis or malignant obstruction from primary airway malignancies (14). Unlike more common causes of acute airway obstruction, which are generally located in the larynx or proximal trachea, these processes are especially challenging in terms of establishing a patent airway through or below the level of obstruction and, as a result, require an alternative surgical approach. We discuss the management of these conditions in a separate 
section of this perspective.

\section{Management of the acute airway-nonsurgical interventions}

Initial management of the acute airway begins with optimizing oxygen delivery. Bag mask ventilation should immediately commence for patients without adequate respirations, with proper maneuvers to align the oral, laryngeal and pharyngeal axes, thereby providing effective breaths (15). Pre-oxygenation prior to elective intubation requires several minutes of $100 \%$ oxygen administration, thereby replacing the nitrogen-laden air in the lung. In emergent intubations, however, several studies have suggested that fewer than one minute of rapid preoxygenation or ten adequate tidal volume breaths achieve similar levels of mean arterial oxygen tension (16).

The mainstay of acute airway management is transoral endotracheal intubation via direct laryngoscopy with simultaneous administration of sedation and paralytics, known as rapid sequence intubation (RSI). RSI has been shown to be successful and limits complications especially in the management of the emergency airway (17). The equipment, medications and training required are readily available in medical centers and studies have shown trainees are able to successfully perform RSI as well as more experienced providers (18).

In those situations where transoral endotracheal intubation is not possible with direct laryngoscopy, indirect laryngoscopy may be employed. Videolaryngoscopy allows for transoral or transnasal visualization of airway structures via fiberoptic videography (19). In a recent Cochrane review comparing direct and videolaryngoscopy for transoral endotracheal intubation, videolaryngoscopy demonstrated fewer failed intubations and improved view of the glottis; however, there were no significant reduction in intubation times, hypoxic events, or mortality (20). Additional benefits of videolaryngoscopy include a quicker learning curve compared to direct laryngoscopy, less required cervical spine manipulation and improved visualization for operators and supervising clinicians. Inherent risks of indirect visualization include potential trauma to oral, pharyngeal and laryngeal soft tissues above the field of view provided by the fiberoptic videography (21). The increasing availability of videolaryngoscopy is coupled with a greater number of traumatic intubations, most commonly throughand-through soft-tissue injuries (22). We recommend that direct laryngoscopy should remain the standard of care and videolaryngoscopy should be used as a first-line adjunct in difficult airways.

\section{Surgical management of the acute airway}

When endotracheal intubation cannot be achieved by direct laryngoscopy and available adjuncts, a surgical airway must be placed. Surgical intubation of the trachea can be accomplished with a variety of techniques, including transtracheal needle ventilation and percutaneous or open tracheostomy.

Transtracheal needle ventilation involves the percutaneous introduction of a large-bore intravenous needle and cannula into the airway with subsequent instillation of oxygen via wall-source, bag-masks, or via high-pressure jet-ventilators (23). While equipment for this approach is minimal, mechanical failures from kinking or dislodgement of the flexible cannula are common. Additionally, oxygenated air instilled through the catheter will flow proximally through the low-resistance upper airway, rather than distally toward the alveoli for gas exchange. Furthermore, unlike cuffed endotracheal or tracheostomy tubes, there is no distal airway protection from upper airway secretions or bleeding. Because this system relies on forced inspiration with passive expiration, there may be subsequent barotrauma and hypercarbia from excessive tidal volumes (24). For these reasons, we do not advocate routine use of transtracheal needle ventilation and recommend the thoracic surgeon employ this technique only when alternative means are not possible.

Cricothyroidotomy requires the sharp incision of the cricothyroid membrane and subsequent intubation with a cuffed endotracheal or tracheostomy tube. In doing so, the airway is patent, the lower airways are protected by the inflatable cuff and adequate gas exchange occurs. It therefore remains the preferred choice of obtaining a surgical airway (25).

The limitations of cricothyroidotomy relate to the knowledge of the surgical anatomy, necessary equipment and potential for injury to surrounding structures. Prospective studies have shown that in non-emergent settings, clinicians, including surgeons, can successfully palpate the cricothyroid membrane in only $70 \%$ of patients, and this number drastically falls to $30 \%$ if the patient is obese (26-29). With the increasing prevalence of portable ultrasonography, studies on the use of ultrasound-guided cricothyroidotomy found no difference in the successful identification of the cricothyroid membrane and other 
critical neck structures (28). Ultrasonography more than doubled the time to successful identification of the cricothyroid membrane. The thoracic surgeon, in this regard, is one of the most experienced clinicians in terms of recognition of surface landmarks and knowledge of underlying neck anatomy.

After successful identification of the surface anatomy, a vertical incision is made in the overlying skin and soft tissue. While a transverse incision is also effective, a vertical incision can be carried cranially or caudally if needed to identify underlying membranous tissue. Proper identification of the midline at the onset of the procedure is paramount for successful cricothyroidotomy. If offcenter, significant bleeding can occur from injury to the infrahyoid muscles, the anterior jugular veins, and the carotid sheath contents. At the time of incising the cricothyroid membrane, a transverse incision is made with care to not incise deeply through the membranous wall of the trachea or esophagus. An audible gush of air can confirm the location. If available, a tracheal hook can be used to lift the cricoid cartilage anteriorly and inferiorly. A Trousseau tracheal dilator is then inserted to dilate the track and a small cuffed endotracheal or tracheostomy tube is inserted. The cuff is insufflated and confirmation of adequate ventilation is performed in standard fashion. We recommend immediate chest radiography to confirm positioning of the endotracheal or tracheostomy tube, as well as urgent surgical consultation for revision to formal tracheostomy within 72 hours (29). The latter is important to minimize long term subglottic tracheal stenosis and tracheomalacia (30).

\section{Surgical cricothyroidotomy versus percutaneous tracheostomy for acute airway management}

There is a growing body of literature describing the use of percutaneous tracheostomy as an alternative method of managing the acute airway $(9,10)$. The procedure involves percutaneous access and passage of a wire into the airway below the vocal cords under direct bronchoscopic visualization with subsequent track dilation and placement of a cuffed tracheostomy tube using Seldinger technique (Supplemental Video).

There are no prospective randomized-controlled trials comparing surgical cricothyroidotomy and percutaneous tracheostomy for the management of the acute airway. However, in retrospective reviews, advocates for percutaneous tracheostomy highlight several benefits (31).
The equipment for percutaneous tracheostomy is packaged as a kit and available in most medical centers. Correct preprocedural identification of the cricothyroid membrane is unnecessary; rather, identification of the upper tracheal rings can be more accurately identified using reliable landmarks, such as the thyroid cartilage and sternal notch. The technical skills required to perform a percutaneous tracheostomy, namely Seldinger technique, are familiar in comparison to surgical cricothyroidotomy and therefore may be more successfully performed by non-surgical clinicians. In comparison to a smaller endotracheal tube placed in surgical cricothyroidotomy, percutaneous tracheostomy allows for a larger inner diameter and shorter length tube, which minimizes airway resistance provides airway access for most diagnostic and therapeutic bronchoscopes. Non-emergent percutaneous tracheostomy is a routine procedure, which makes it more like likely to be performed successfully in an emergent setting. Many clinicians, including thoracic surgeons, have never performed a surgical cricothyroidotomy, which makes the index case even more challenging. Lastly, unlike after surgical cricothyroidotomy, conversion to formal tracheostomy is not required.

The thoracic surgeon should also be aware of the drawbacks to percutaneous tracheostomy as a technique for acute airway management. If a percutaneous tracheostomy kit is unavailable, it is unsafe and time-consuming to piece together a kit from separate equipment in an emergency. While surgical cricothyroidotomy also requires certain equipment, it can be performed with only a scalpel and endotracheal tube. In addition, percutaneous tracheostomy is most safely completed under bronchoscopy, which ensures that the guidewire, tracheal dilator and tracheostomy are passed into the airway. Without bronchoscopy, there is risk of creating false passages cranially, caudally and laterally within the neck, with subsequent damage to surrounding structures (32). Even without bronchoscopy, percutaneous tracheostomy placement takes longer than surgical cricothyroidotomy. In human cadaver models and in the hands of inexperienced clinicians, percutaneous tracheostomy takes nearly twice as long in comparison to surgical cricothyroidotomy (135 versus 78 seconds) (33). Furthermore, operators were less likely to be successful with percutaneous tracheostomy. Failed attempts may complicate subsequent surgical cricothyroidotomy and necessitate a more extensive dissection.

We believe that surgical cricothyroidotomy remains the gold standard technique for establishing an airway after 
failed endotracheal intubation. The data comparing surgical cricothyroidotomy and percutaneous tracheostomy are lacking and we recognize that based on operator experience, patient stability and equipment availability, percutaneous tracheostomy may be a preferred approach. The thoracic surgeon should be aware of the technique, risks and benefits of the procedure and outcomes of both surgical cricothyroidotomy and percutaneous tracheostomy.

\section{Prevention: tracheal obstruction and the thoracic surgeon}

The thoracic surgeon routinely encounters patients at risk of developing an acute airway obstruction, and is responsible for surveilling these patients to prevent fatal complications. The most common non-neoplastic cause of airway obstruction is post-intubation tracheal stenosis (2). This may develop from circumferential stenosis from chronic inflammation from inflatable cuffs, or anterior scarring from a tracheostomy stoma. Neoplastic obstruction can occur from primary airway tumors or external compressing tumors. As mentioned, these lesions may occur anywhere along the tracheobronchial tree and therefore patient presentation and surgical management vary on location.

Patients with chronic partial tracheal obstruction require frequent surveillance with history and physical examination, imaging and operative evaluation. For symptomatic patients, admission to a monitored inpatient unit is recommended. Administration of humidified oxygen and helium-oxygen mixtures, the latter of which has a lower density than nitrogen-rich air and therefore optimizes laminar flow of oxygen to the alveoli, may improve symptoms and prevent intubation (34). Blood tests should be ordered to investigate for biochemical derangements or acid-base disturbances that may alter respiratory drive. Imaging includes plain chest radiography, which may importantly determine the level of obstruction by identifying a column of air within the airway. Computed tomography (CT) of the neck and chest using intravenous contrast in inspiratory and expiratory phases is the gold standard imaging for suspected tracheal obstruction. This modality can characterize the location and features of the obstruction, as well as demonstrate any dynamic causes of obstruction (35).

If warranted, management begins with rigid bronchoscopy for further evaluation of the cause of obstruction and potential treatment based on the underlying pathology. Coordination with an anesthesiologist experienced with use of inhaled anesthesia to avoid apnea is recommended. Necessary equipment includes Jackson or Storz rigid bronchoscopes with ventilating side arms, Jackson-Pilling dilating esophageal bougies, biopsy forceps and a 0 -degree Hopkins telescope (36). Rigid bronchoscopy begins with careful introduction of the bronchoscope into the trachea under direct visualization. Care is made to complete a thorough mucosal evaluation. Routine measurements are taken to determine the level of the lesion. It is important to not pass beyond a narrow stenosis to avoid bleeding from the lesion or trauma to distal healthy trachea. Biopsy forceps are used to obtain a tissue diagnosis. For non-neoplastic lesions, dilation is the mainstay of treatment. Circumferential lesions tend to have a better response to dilation in comparison to focal areas of granulation tissue, which may be amenable to removal with biopsy forceps or laser ablation (37). Neoplastic lesions should be further evaluated with primary staging studies and a metastatic evaluation; if symptoms mandate treatment, gentle dilatation, tracheal stenting, or placement of a T-tube may be employed to provide a safe interval until a definitive operation may be performed (38).

\section{Conclusions}

Acute airway management remains a prevalent and clinically important issue for all health care providers. The history of acute airway management underscores key milestones and innovations in airway surgery, anesthesia, pharmacology and technology. Understanding this progress allows us to pay homage to those pioneers in each of these fields.

Acute airway management begins with prompt recognition of the clinical problem, assessment of patient stability and emergent airway control. With the review of the non-surgical and operative techniques in tracheal intubation, as well as available adjuncts, we believe the airway control is achieved with transoral endotracheal intubation under direct laryngoscopy with surgical cricothyroidotomy for failed attempts in the unstable patient. We believe this is the safest and most effective management strategy, although we recognize that provider experience, technology and resource availability may allow for alternative algorithms.

The thoracic surgeon has an especially unique role in the management of the acute airway, both in regard to a more experienced background in surface and tracheal anatomy as well as in prevention and close follow-up in high risk patients with partial airway obstructions. A routine 
review of available management strategies and emerging technology is recommended to continue to provide patients with excellent and evidence-based care.

\section{Acknowledgements}

None.

\section{Footnote}

Conflicts of Interest: The authors have no conflicts of interest to declare.

\section{References}

1. Szmuk P, Ezri T, Evron S, et al. A brief history of tracheostomy and tracheal intubation, from the Bronze Age to the Space Age. Intensive Care Med 2008 34:222-8.

2. Wain JC. Postintubation tracheal stenosis. Chest Surg Clin N Am 2003;13:231-46.

3. Pearce JM. Armand Trousseau--some of his contributions to neurology. J Hist Neurosci 2002;11:125-35.

4. Ezri T, Evron S, Hadad H, et al. Tracheostomy and endotracheal intubation: a short history. Harefuah 2005;144:891-3, 908.

5. Zeitels SM. Chevalier Jackson's contributions to direct laryngoscopy. J Voice 1998;12:1-6.

6. Burkle CM, Zepeda FA, Bacon DR, et al. A historical perspective on use of the laryngoscope as a tool in anesthesiology. Anesthesiology 2004;100:1003-6.

7. Robinson DH, Toledo AH. Historical development of modern anesthesia. J Invest Surg 2012;25:141-9.

8. Raghavendra T. Neuromuscular blocking drugs: discovery and development. J R Soc Med 2002;95:363-7.

9. Brass P, Hellmich M, Ladra A, et al. Percutaneous techniques versus surgical techniques for tracheostomy. Cochrane Database Syst Rev 2016;7:CD008045.

10. Ciaglia P, Firsching R, Syniec C. Elective percutaneous dilatational tracheostomy. A new simple bedside procedure; preliminary report. Chest 1985;87:715-9.

11. Linscott MS, Horton WC. Management of upper airway obstruction. Otolaryngol Clin North Am 1979;12:351-73.

12. LoVerde D, Iweala OI, Eginli A, et al. Anaphylaxis. Chest 2018;153:528-43.

13. Pardal-Refoyo JL, Ochoa-Sangrador C. Bilateral recurrent laryngeal nerve injury in total thyroidectomy with or without intraoperative neuromonitoring. Systematic review and meta-analysis. Acta Otorrinolaringol Esp
2016;67:66-74.

14. Ernst A, Feller-Kopman D, Becker HD, et al. Central airway obstruction. Am J Respir Crit Care Med 2004;169:1278-97.

15. Janssens M, Hartstein G. Management of difficult intubation. Eur J Anaesthesiol 2001;18:3-12.

16. Gold MI, Duarte I, Muravchick S. Arterial oxygenation in conscious patients after 5 minutes and after 30 seconds of oxygen breathing. Anesth Analg 1981;60:313-5.

17. Okubo M, Gibo K, Hagiwara Y, et al. The effectiveness of rapid sequence intubation (RSI) versus non-RSI in emergency department: an analysis of multicenter prospective observational study. Int J Emerg Med 2017;10:1.

18. Sagarin MJ, Barton ED, Chng YM, et al. Airway management by US and Canadian emergency medicine residents: a multicenter analysis of more than 6,000 endotracheal intubation attempts. Ann Emerg Med 2005;46:328-36.

19. Butler KH, Clyne B. Management of the difficult airway: alternative airway techniques and adjuncts. Emerg Med Clin North Am 2003;21:259-89.

20. Lewis SR, Butler AR, Parker J, et al. Videolaryngoscopy versus direct laryngoscopy for adult patients requiring tracheal intubation. Cochrane Database Syst Rev 2016;11:CD011136.

21. Chemsian R, Bhananker S, Ramaiah R. Videolaryngoscopy. Int J Crit Illn Inj Sci 2014;4:35-41.

22. Greer D, Marshall KE. Bevans S, et al. Review of videolaryngoscopy pharyngeal wall injuries. Laryngoscope 2017;127:349-53.

23. Ihra G, Gockner G, Kashanipour A, et al. Highfrequency jet ventilation in European and North American institutions: developments and clinical practice. Eur J Anaesthesiol 2000;17:418-30.

24. Law JA, Broemling N, Cooper RM, et al. The difficult airway with recommendations for management-part 1--difficult tracheal intubation encountered in an unconscious/induced patient. Can J Anaesth 2013;60:1089-118.

25. Brantigan CO, Grow JB. Cricothyroidotomy: elective use in respiratory problems requiring tracheotomy. J Thorac Cardiovasc Surg 1976;71:72-81.

26. Hiller KN, Karni RJ, Cai C, et al. Comparing success rates of anesthesia providers versus trauma surgeons in their use of palpation to identify the cricothyroid membrane in female subjects: a prospective observational study. Can J Anaesth 2016;63:807-17. 
27. You-Ten KE, Desai D, Postonogova T, et al. Accuracy of conventional digital palpation and ultrasound of the cricothyroid membrane in obese women in labour, Anaesthesia 2015;70:1230-4.

28. Yıldiz G, Goksu E, Senfer A, et al. Comparison of ultrasonography and surface landmarks in detecting the localization for cricothyroidotomy. Am J Emerg Med 2016;34:254-6.

29. Hsiao J, Pacheco-Fowler V. Videos in clinical medicine. Cricothyroidotomy. N Engl J Med 2008;358:e25.

30. Sise MJ, Shackford SR, Cruickshank JC, et al. Cricothyroidotomy for long-term tracheal access. A prospective analysis of morbidity and mortality in 76 patients. Ann Surg 1984;200:13-7.

31. Davidson SB, Blostein PA, Walsh J, et al. Percutaneous tracheostomy: a new approach to the emergency airway., J. Trauma Acute Care Surg 2012;73:S83-8.

32. Cipriano A, Mao ML, Hon HH, et al. An overview of complications associated with open and percutaneous

Cite this article as: Panda N, Donahue DM. Acute airway management. Ann Cardiothorac Surg 2018;7(2):266-272. doi: 10.21037/acs.2018.01.15 tracheostomy procedures. Int J Crit Illn Inj Sci 2015;5:179-88.

33. Schober P, Hegemann MC, Schwarte LA, et al. Emergency cricothyrotomy-a comparative study of different techniques in human cadavers. Resuscitation 2009;80:204-9.

34. McGarvey JM, Pollack CV. Heliox in airway management. Emerg Med Clin North Am 2008;26:905-20, viii.

35. Aquino SL, Shepard JA, Ginns LC, et al. Acquired tracheomalacia: detection by expiratory CT scan. J Comput Assist Tomogr 2001;25:394-9.

36. Grillo HC. Surgery of the Trachea and Bronchi. 1st edition. People's Medical Publishing House--USA LTD, 2003.

37. Grillo HC, Donahue DM. Post intubation tracheal stenosis. Semin Thorac Cardiovasc Surg 1996;8:370-80.

38. Gaissert HA, Grillo HC, Mathisen DJ, et al. Temporary and permanent restoration of airway continuity with the tracheal T-tube. J Thorac Cardiovasc Surg 1994;107:600-6. 